International Journal of Computer Networks \& Communications (IJCNC) Vol.8, No.3, May 2016

\title{
JOINT-DESIGN OF LINK-ADAPTIVE MODULATION AND CODING WITH ADAPTIVE ARQ FOR COOPERATIVE AMPLIFY AND FORWARD RELAYING SYSTEM
}

\author{
Bhuvan Modi, O. Olabiyi and A. Annamalai \\ Center of Excellence for Communication Systems Technology Research, Department of \\ Electrical and Computer Engineering, Prairie View A \& M University, TX 77446 United \\ States of America
}

\begin{abstract}
This paper analyzes the efficiency of a joint-design of an adaptive modulation and coding (AMC) at the physical (PHY) layer with an adaptive $R^{\max }$-truncated selective-repeat automatic repeat request (ARQ) protocol at the medium access control (MAC) layer to maximize the throughput of cooperative nonregenerative relay networks under prescribed delay and/or error performance constraints. Particularly, we generalize the existing design model/results for cross-layer combining of AMC along with truncated ARQ in non-cooperative diversity networks in three-folds: (i) extension of the cross-layer PHY/MAC design or optimization to cooperative diversity systems; (ii) generalization/unification of analytical expressions for various network performance metrics to generalized block fading channels with independent but nonidentically distributed (i.n.d) fading statistics among the spatially distributed nodes; (iii) analysis of the effectiveness of joint-adaptation of the maximum retransmission limit $R^{\text {max }}$ of $A R Q$ protocol and cooperative diversity order $N$ for delay-insensitive applications. Our insightful numerical results reveal that the average throughput can be increased significantly by judiciously combining two additional degrees of freedom $\left(N\right.$ and $\left.R^{\max }\right)$ that are available in cooperative amplify-and-forward $(C A F)$ relay networks besides employing AMC at the PHY layer, especially in the most challenging low signal-to-noise ratio (SNR) regime.
\end{abstract}

\section{KEYWORDS}

cross-layer design, adaptive retransmission, cooperative relay diversity, adaptive modulation and coding

\section{Motivation}

It is well-known that the link adaptation techniques (e.g., adaptive modulation/coding) could dramatically enhance the spectral utilization efficiency of wireless networks that employ "fixedtransmission" methods. But to improve the transmission reliability/robustness at the physical (PHY) layer, one has to either increase the transmit power (thereby, decreasing the battery-life) or to reduce the transmission rate (e.g., by selecting a smaller constellation size or decreasing the code rate of forward error correction coding). Additionally, spatial/polarization diversity solutions at the physical layer (by employing multiple antenna elements at the transmitter and/or receiver) may not be reasonable on small-sized handheld portable devices or sensor nodes. 
An alternative way to mitigate the deleterious effects of a multipath fading is to exploit "diversity" mechanisms at higher layers of the protocol stack. For instance, ARQ is an effective strategy to achieve a high reliability of packet transmissions at the data link layer (especially in slowly time varying channels) and unlike FEC, the redundancy (packet retransmissions) are only introduced, when necessary.

Similarly, the number of collaborating nodes in a CAF relay network (i.e., distributed spatial diversity order) could be increased to satisfy the prescribed average packet error rate constraint (but the reliability improvement is attained at the expense of the network capacity owing to the half-duplex operation of CAF relay networks, although this technique could overcome the practical implementation issue of packing multiple antenna elements on small-sized sensor nodes).

Instead of considering AMC at the PHY layer, ARQ at the data link layer, and cooperative diversity at the network layer separately in this article, we pursue a cross-layer design that combines these three layers judiciously to maximize the spectral efficiency or throughput subject to delay and/or error performance constraints. Cross-layer design approach breaks away from conventional network design, where each and every layer of the protocol stack is optimized and operates independently. In particular, we exploit the channel knowledge at transmitter and explore the potential synergies between different protocol layers to maximize the end-to-end throughput while satisfying the prescribed delay and average packet error rate (APER) constraints. For example, by achieving a higher packet success probability with the help of cooperative diversity and ARQ, the stringent error control requirement is improved for the AMC at the PHY layer. This enables a considerable spectral efficiency gain especially in the low SNR regime. Given the maximum allowable number of retransmissions $R^{\max }$ (that depends on the delay constraint) in a CAF relay network, we design AMC transmissions that guarantee the required APER performance. The benefits of adapting $R^{\mathrm{max}}$ and the number of cooperating relay nodes are also investigated.

\subsection{Literature Review/Prior Work}

While the literature on performance analysis of non-adaptive (i.e., fixed-rate and/or fixed-power) cooperative diversity systems and adaptive transmission techniques for non-cooperative wireless networks are quite extensive that span over four decades, most prior focused only on the improvement of the link layer performance. The art of adaptive link layer for cooperative wireless networks especially in a cross-layer design framework is still in its infancy. For instance, in [1][3] (and references therein), the authors have studied extensively the design and implementation of AM and coding at the PHY layer, wherein the transmission rates are matched to the timevarying channel conditions in a non-cooperative wireless system. Author in ref. [4] investigates the efficiency of a truncated ARQ protocol scheme for a cooperative amplify-and-forward system with fixed modulation. Authors in ref. [5]-[9] have considered Adaptive Modulation and/or optimal power allocation amongst collaborating nodes in cooperative relay systems. Whereas authors in ref. [10] studied the effectiveness of cross-layer combining of the ARQ and the AMC for non-cooperative diversity systems in a Nakagami-m fading channel. Authors in [11] analyzed the performance of a cross layer design in terms of spectral efficiency, which combines cooperative diversity with truncated ARQ in Ad-hoc wireless networks, but without link adaptation in the Rayleigh fading channel. Ref. [12],[13] studied the spectral efficiency analysis of Joint AMC and Cooperative ARQ for a single incremental relay in the Rayleigh fading channel. In [14], authors considered a cross-layer combination of a cooperative hybrid ARQ with adaptive modulation in wireless ad-hoc networks by assuming a single retransmission request under the Rayleigh fading environment. 
Motivated by above observations/discussions, our contributions in this paper can be summarized as follows:

1. Motivated by the appreciable improvement in the data link layer throughput by judiciously combining a truncated ARQ protocol with adaptive modulation and coding (AMC) over the simple concatenation of ARQ to fixed modulation/coding schemes, we consider a design methodology similar to [10] but with two additional degrees of freedom for providing the desired level of rate-reliability trade-off via cooperative diversity and adaptive $R^{\max }$ strategy. In addition, we developed a novel unified analytical framework (based on the marginal MGF of the end-to-end SNR) to compute the average spectral efficiency, outage probability and APER performance metrics over fading channels (viz., since the MGF of the end-to-end SNR is much easier to compute and/or readily available for CAF relay networks compared to its probability density function, while the marginal MGF can be computed efficiently using this MGF in conjunction with Fixed-Talbot method [15]). Our proposed mathematical framework is satisfactorily general to exemplify the performance of adaptive-link non-regenerative relay networks over a extensive range of fading distributions (i.e., it is not only restricted to the Rayleigh or independent identically distributed (i.i.d) Nakagami-m fading channel) with independent and non-identically distributed (i.n.d) fading statistics across the spatially distributed diversity paths and can be efficiently apply to the wireless system composed of large number of relays.

2. Moreover, we propose an interesting approach for maximization of throughput by joint adaptation of two parameters, one with cooperative diversity order $N$ and second with adaptive $R^{\max }$ scheme (see Fig. 8). To the best of our knowledge a similar approach which focuses on throughput optimization by jointly adaptation of both $N$ and $R^{\max }$ has not been considered in the previous literature.

The remainder of this paper is organized as follows. System model is discussed in section 2. We develop the cross-layer design in Section 3, by combining AMC at the physical layer with adaptive ARQ at the data link layer for CAF relay networks. Numerical findings are presented in Section 4. Our conclusions are given in Section 5.

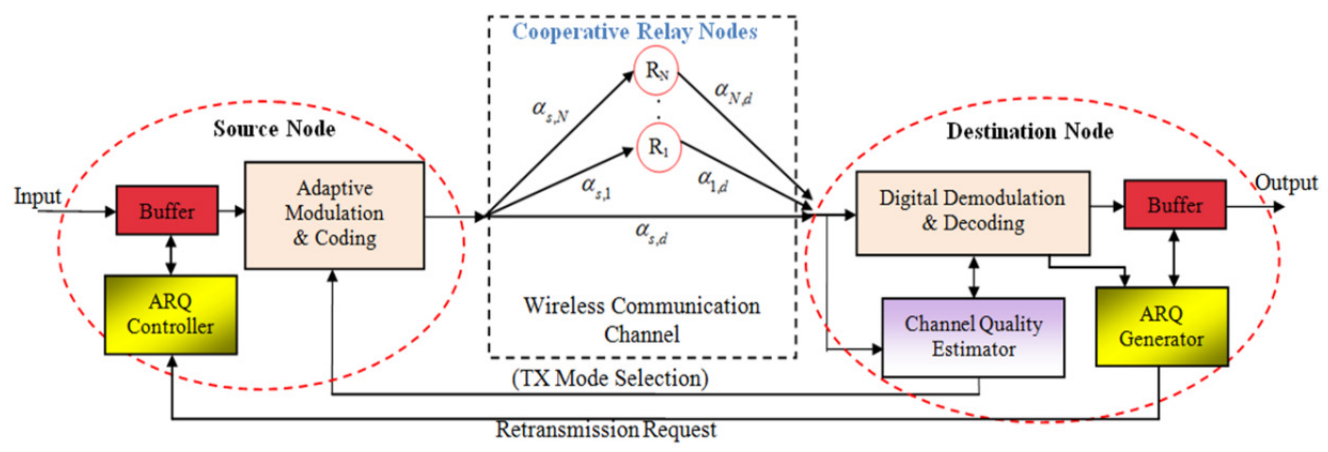

Figure 1. System Model: Link-adaptive cooperative diversity system with ARQ technique 
International Journal of Computer Networks \& Communications (IJCNC) Vol.8, No.3, May 2016

\section{SYSTEM MODEL}

\subsection{CoOperative Diversity MODEL}

Figure 1 shows combined link-adaptive and ARQ based cooperative diversity system with a source node $S$ communicates with a destination node $D$ via a direct-link and through $N$ amplifyand-forward relays, $R_{i}, i \in\{1,2, \ldots, N\}$, in two transmission phases. During the initial Phase I, $S$ broadcasts signal $x$ to $D$ and to the relays $R_{i}$, where channel fading coefficients between $S$ and $D$, $S$ and the $i$-th relay node $R_{i}, R_{i}$ and $D$ are denoted by $\alpha_{s, d}, \alpha_{s, i}$ and $\alpha_{i, d}$, respectively. In the second segment of cooperation, each of the $N$ relays re-transmits the received signal after amplification via orthogonal transmissions (using TDMA in a round-robin fashion and/or FDMA). If a maximum ratio combiner (MRC) process is deployed at the destination node $D$ to coherently merge all the signals received during these two transmission phases, the effective end-to-end SNR is given by [17],[16]

$$
\gamma=\gamma_{s, d}+\sum_{i=1}^{N} \frac{\gamma_{s, i} \gamma_{i, d}}{1+\gamma_{s, i}+\gamma_{i, d}} \cong \gamma_{s, d}+\sum_{i=1}^{N} \gamma_{i}=\gamma_{s, d}+\sum_{i=1}^{N} \frac{\gamma_{s, i} \gamma_{i, d}}{\gamma_{s, i}+\gamma_{i, d}}
$$

where $\gamma_{i}=\gamma_{s, i} \gamma_{i, d} / \gamma_{s, i}+\gamma_{i, d}, \gamma_{a, b}=\left|\alpha_{a, b}\right|^{2} E_{s} / N_{o}$ corresponds to the instantaneous SNRs of link $a-b$, $E_{s}$ represents the average symbol energy and $N_{0}$ denotes the noise variance.

The approximation of (1) is obtained by recognizing that the instantaneous SNR of a two hops path can be accurately estimated to be the harmonic mean of individual link SNRs especially at moderate/high SNR regimes [18]. Hence, it is straight-forward to show that the MGF of $\gamma$ is given by [18]

$$
\phi_{\gamma}(s)=\phi_{\gamma_{s, d}}(s) \prod_{i=1}^{N} \phi_{\gamma_{i}}(s)
$$

where $\phi_{\gamma_{s, d}}(s)$ and $\phi_{\gamma_{i}}(s)$ denote the MGF of SNR of the S-D link and two-hops relayed paths, respectively.

\begin{tabular}{|c|c|c|c|c|c|c|c|}
\hline \multicolumn{8}{|c|}{ Different Transmission Modes in TM1 with Uncoded $M_{n}$-QAM Modulation } \\
\hline Mode & Mode 1 & Mode 2 & Mode 3 & Mode 4 & Mode 5 & Mode 6 & Mode 7 \\
\hline Modulation & BPSK & QPSK & 8-QAM & 16-QAM & 32-QAM & 64-QAM & 128-QAM \\
\hline Rates (bits/sym.) & 1 & 2 & 3 & 4 & 5 & 6 & 7 \\
\hline$a_{n}$ & 67.7328 & 73.8279 & 58.7332 & 55.9137 & 50.0552 & 42.5594 & 40.2559 \\
\hline$g_{n}$ & 0.9819 & 0.4945 & 0.1641 & 0.0989 & 0.0381 & 0.0235 & 0.0094 \\
\hline$b_{n}(\mathrm{~dB})$ & 6.3281 & 9.3945 & 13.9470 & 16.0938 & 20.1103 & 22.0340 & 25.9677 \\
\hline \multicolumn{8}{|c|}{ Different Transmission Modes in TM2 with Convolutionally coded $M_{n}$-QAM Modulation } \\
\hline \multicolumn{2}{|c|}{ Mode } & Mode 1 & Mode 2 & Mode 3 & Mode 4 & Mode 5 & Mode 6 \\
\hline \multicolumn{2}{|c|}{ Modulation } & BPSK & QPSK & QPSK & 16-QAM & 16-QAM & 64-QAM \\
\hline \multicolumn{2}{|c|}{ Coding Rate $R_{c}$} & $1 / 2$ & $1 / 2$ & $3 / 4$ & $9 / 16$ & $3 / 4$ & $3 / 4$ \\
\hline \multicolumn{2}{|c|}{ Rates (bits/sym.) } & 0.50 & 1.00 & 1.50 & 2.25 & 3.00 & 4.50 \\
\hline \multicolumn{2}{|l|}{$a_{n}$} & 274.7229 & 90.2514 & 67.6181 & 50.1222 & 53.3987 & 35.3508 \\
\hline \multicolumn{2}{|l|}{$g_{n}$} & 7.9932 & 3.4998 & 1.6883 & 0.6644 & 0.3756 & 0.0900 \\
\hline \multicolumn{2}{|c|}{$b_{n}(\mathrm{~dB})$} & -1.5331 & 1.0942 & 3.9722 & 7.7021 & 10.2488 & 15.9784 \\
\hline
\end{tabular}

Table I 


\subsection{AdAPTIVe Modulation ANd CODing (AMC) SCHEME}

Suppose that the multiple transmission modes are available at the PHY layer, and each associated with a specific AMC scheme. In practice, link-adaptation is performed at the frame level (which is the processing unit at the PHY layer) and the AMC controller at the transmitter (i.e., source node $S$ ) selects a particular mode for transmission based on the feedback of channel side information (e.g., effective SNR) acquired by the destination node D. But APER evaluation (required for MAC layer throughput calculation) through the average bit error rate using $\overline{P_{B}}=1-\left(1-\bar{P}_{b}\right)^{N_{p}}$ may not be always accurate especially for higher order constellations (since information bits in a symbol incur different error probabilities) and coded transmissions over slow fading channels (since bit errors are not uncorrelated). Moreover, this form does not provide the averaging problem over the fading SNR density function that arises in the performance evaluation of AMC systems. In this article, we will utilize an exponential-type approximation for the instantaneous packet error rate (PER) provided in [10]. At the physical layer, following two sets of transmission modes are considered (listed in Table $\mathrm{I}^{1}$ ): TM1- is uncoded, with $M_{n}$-ary rectangular/square QAM modes (where $M_{n}=2^{n}, n=1,2,3,4 \ldots 7$ ) and TM2-is convolutionally coded $M_{n}$-ary rectangular or square QAM modes.

\subsection{Selective-Repeat ArQ Protocol Scheme}

The selective-repeat ARQ protocol is implemented at the data link layer with a retransmission limit $R^{\max }$ (while only finite delays and buffer sizes can be afforded in practice), and hence errorfree delivery is not guaranteed. The value of $R^{\max }$ can be determined by dividing the maximum permissible network delay by the round-trip delay required for each retransmission. If a packet is not received correctly after $R^{\max }$ retransmissions, it will be dropped and we declare packet loss. In our cross-layer design, our design objective is to select an appropriate modulation scheme that ensures that the packet loss after $R^{\max }$ retransmissions is no larger than the target packet loss probability, $P_{\text {loss }}$.

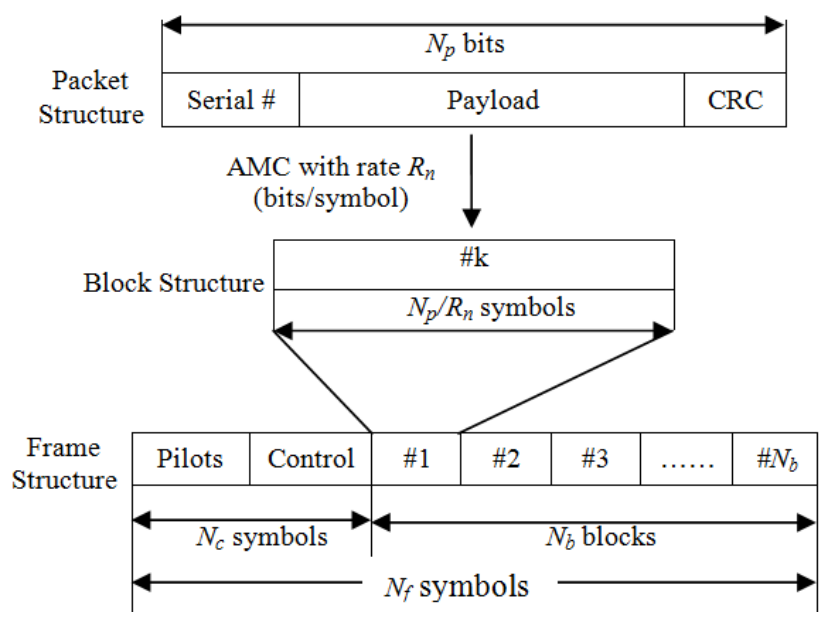

Figure 2. Illustration of packet and frame structures

\footnotetext{
${ }^{1}$ The coefficients in Table I were obtained in [8] for packet size $N_{p}=1080$ bits (chosen arbitrarily). For other values of the packet lengths, new optimized coefficients can be obtained in a similar fashion.
} 
The packet and frame structures are depicted in Fig. 2. It is considered that, at the data link layer, each packet consists of $N_{p}$ bits that, contains a payload, serial number, and cyclic redundancy check (CRC) bits for error detection. Each packet is mapped into a block consisting of $N_{p} / R_{n}$ symbols where $R_{n}$ denotes the rate or number of bits/symbol in mode $n$, while each frame at the PHY layer contains $N_{b}$ blocks (depends on the chosen modulation) along with $N_{c}$ pilot symbols and control bits (i.e., each frame consists of $N_{f}=N_{c}+N_{b} N_{p} / R_{n}$ symbols).

\section{Cross-Layer Combining Of AMC With Truncated ARQ Over FADING CHANNEL}

In this section, we discuss our cross-layer design which combines AMC at the PHY layer with an adaptive ARQ at the data link layer for multi-relay two-hop CSI-assisted CAF networks. We also outline the development of our unified expressions (i.e., that involves computing the difference between two "CDF" terms as in (6) in conjunction with closed-form formulas for the MGF of $\gamma_{i}$ or $\left.\tilde{\gamma}_{i}=\min \left(\gamma_{s, i}, \gamma_{i, d}\right)\right)$ for calculating the APER, average spectral efficiency and outage probability performance metrics. Moreover, extension this to blind relays and cooperative decode-andforward relay system is quite straight-forward [19].

\subsection{Performance ReQuirement At The PHY Layer}

We first determine what should be the instantaneous PER (without retransmissions) at the PHY layer in order to satisfy the requirement that the packet loss probability after $R^{\max }$ retransmissions is no larger than $P_{\text {loss? }}$ ? Assume that instantaneous PER is guaranteed to be no greater than $P_{0}$, for each chosen adaptive modulation and coding transmission mode at the PHY layer. Thus, it is simple to show that

$$
P_{0} \leq P_{\text {loss }}^{1 /\left(R^{\max }+1\right)}=P_{\text {target }}
$$

To satisfy the PER upper bound as in eq. (3), if we design AMC at the PHY layer and employ $R^{\max }$ truncated ARQ at the data link layer, then both delay and error performance constraints will be satisfied simultaneously. In the following subsections, we will consider the joint-design of AMC in a CAF network with $N$ cooperating relays to maximize spectral efficiency while satisfying the error performance (3).

\subsection{AMC Design At The PHY LAYER}

Let us assume that the sum of transmit powers from all cooperating nodes is constant and the range of effective end-to-end SNR (1) is partitioned into $T+1$ non-overlapping consecutive intervals with boundary points denoted as $\left\{\lambda_{n}\right\}_{n=0}^{T+1}$. For instance, mode $n$ is chosen when $\gamma \in\left[\lambda_{n}, \lambda_{n+1}\right)$ and the transmission will be ceased (no payload bits will be sent) when $0 \leq \gamma<\lambda_{1}$ to avoid deep channel fades. Remaining task now is to determine the boundary points (switching SNR thresholds) required to attain $P_{\text {target }}$.

Since our system uses packets as processing units, we rely on the following exponential-type PER approximation to simplify the AMC design [10], viz.,

$$
P E R_{n}(\gamma) \approx\left\{\begin{array}{cl}
1 & \text { if } 0<\gamma<b_{n}, \\
a_{n} \exp \left(-g_{n} \gamma\right) & \text { if } \gamma \geq b_{n}
\end{array}\right.
$$


where subscript $n$ denotes the transmission mode index, and constants $a_{n}, g_{n}$ and $b_{n}$ are summarized in Table I. Inverting (4), we obtain the minimum SNR thresholds required to achieve $P_{\text {target }}$ as

$$
\begin{gathered}
\lambda_{n}=\left(1 / g_{n}\right) \ln \left(a_{n} / P_{\text {target }}\right), n=1,2, \ldots \ldots ., T \\
\lambda_{0}=0 \text { and } \lambda_{T+1}=+\infty .
\end{gathered}
$$

\subsection{Outage Probability}

When the total received SNR falls below the region boundary threshold $\lambda_{1}\left(\lambda_{1}\right.$ is obtained by substituting $a_{1}$ and $g_{1}$ from Table I in (5)), the source node $\mathrm{S}$ ceases transmission, because the prescribed target PER cannot be satisfied even with the smallest constellation size. The Probability of such an outage event is given by $P_{\text {out }}=F_{\gamma}\left(\lambda_{1}\right)$, where the CDF term can be evaluated efficiently using [15], viz.,

$$
F_{X}(x) \cong \frac{1}{2 Z} \phi_{X}(r) e^{r x}+\frac{r}{Z} \sum_{k=1}^{Z-1} \operatorname{Re}\left\{\frac{1+j \sigma\left(\theta_{k}\right)}{s\left(\theta_{k}\right)} e^{x s\left(\theta_{k}\right)} \phi_{X}\left(s\left(\theta_{k}\right)\right)\right\}
$$

where $\quad r=2 Z /(5 x), \theta_{k}=k \pi / Z, \sigma\left(\theta_{k}\right)=\theta_{k}+\left(\theta_{k} \cot \left(\theta_{k}\right)-1\right) \cot \left(\theta_{k}\right), \quad s\left(\theta_{k}\right)=r \theta_{k}\left(j+\cot \left(\theta_{k}\right)\right), \quad$ and positive integer $Z$ can be chosen appropriately to achieve the desired accuracy. Once the exact, bound or approximate MGF of $\gamma_{i}$ is found, we can easily compute its cumulative distribution function (CDF) efficiently with the aid of multi-precision Laplace inversion formula from (6).

\subsection{Average Packet Error Probability}

The APER for the AMC scheme can be calculated as the ratio of average number of incorrectly received packets over the total average number of transmitted packets [1, eq. (35)], viz.,

$$
P_{e}=\sum_{n=1}^{T} R_{n} P_{n} \overline{P E R_{n}} / \sum_{n=1}^{T} R_{n} P_{n}
$$

where $P_{n}$ denotes the probability of transmitting in mode $n$ (i.e., $\lambda_{n} \leq \gamma<\lambda_{n+1}$ ),

$$
P_{n}=\int_{\lambda_{n}}^{\lambda_{n+1}} f_{\gamma}(\gamma) d \gamma=F_{\gamma}\left(\lambda_{n+1}\right)-F_{\gamma}\left(\lambda_{n}\right)
$$

and $\overline{P E R_{n}}$ represents the mode $n$ APER, and is presented as

$$
\begin{aligned}
\overline{P E R_{n}} & =\frac{1}{P_{n}} \int_{\lambda_{n}}^{\lambda_{n+1}} a_{n} \exp \left(-g_{n} \gamma\right) f_{\gamma}(\gamma) d \gamma \\
& =\left(a_{n} / P_{n}\right)\left[\phi_{\gamma}\left(g_{n}, \lambda_{n}\right)-\phi_{\gamma}\left(g_{n}, \lambda_{n+1}\right)\right]
\end{aligned}
$$

Here, $\phi_{\gamma}(\beta, \alpha)=\int_{\alpha}^{\infty} e^{-\beta \gamma} f_{\gamma}(\gamma) d \gamma$ corresponds to the marginal MGF of the end-to-end SNR as shown in (1). In general, the computation of the marginal MGF is not a trivial task for CAF relay networks.

This may be attributed to the difficulty in deriving the PDF of fading channel SNR. In our work, we circumvent this difficulty by exploiting the results in [20, Appendix B] to compute the marginal MGF via a Laplace inversion of an auxiliary MGF function, viz., 


$$
\overline{P E R_{n}}=\left(a_{n} / P_{n}\right)\left[F_{\hat{\gamma}}\left(\lambda_{n+1}\right)-F_{\hat{\gamma}}\left(\lambda_{n}\right)\right]
$$

where $F_{\hat{\gamma}}(x)$ in (10) can be evaluated efficiently using (6) in conjunction with the "MGF" of an auxiliary function (i.e., $\phi_{\hat{\gamma}}(s)=\phi_{\gamma}\left(s+g_{n}\right)$ ). Furthermore, for cooperative amplify-and-forward case in contrast to the development in [8], our solution in (10) is sufficiently general to characterize the APER performance of transmission mode $n$ over a wide range of fading environments (i.e., given that the marginal MGF for a single channel reception can be expressed in closed-form for any number of stochastic channel models including Nakagami-m, Rice and Nakagami-q channels [21, Appendix B]). It is also important to note that the APER of AMC (7) will always be lower than $P_{\text {target }}$ since the instantaneous PER is upper bounded by $P_{\text {target }}$. To reduce this gap and to further improve the spectral efficiency, an adaptive switching SNR threshold scheme can be designed (instead of using fixed switching SNR thresholds from (5)).

\subsection{AVerage SPECTRAL EFFicienCy}

For specific mode $n$, each transmitted symbol of the adaptive modulation and coding method will carry $R_{n}=\log _{2}\left(M_{n}\right)$ information bits for mode adhering to a $\mathrm{M}_{\mathrm{n}}$-QAM constellation and a rate $R_{c}$ FEC code. For TM1, we set $R_{c}=1$. Suppose that a Nyquist pulse shaping filter with bandwidth $B$ $=1 / T_{s}$, where $T_{s}$ denotes the symbol period, then the mean spectral efficiency (bits per unit bandwidth) at the Phy layer (without taking into account possible packet retransmissions) for a non-regenerative network with $N$ cooperating relays is given by [6]

$$
S_{e, P H Y}=\frac{1}{N+1} \sum_{n=1}^{T} R_{n} P_{n}
$$

For a truncated ARQ protocol which is implemented at the data link layer, packets received in error may be retransmitted up to a maximum of $R^{\max }$ times. Thus the average number of transmissions per packet can be shown as [22, p. 397]

$$
\bar{N}\left(P_{e}, R^{\max }\right)=1+P_{e}+P_{e}^{2}+\ldots .+P_{e}^{R^{\max }}=\left(1-P_{e}^{R^{\max }+1}\right) /\left(1-P_{e}\right),
$$

where $P_{e}$ is defined in (7), and the overall average spectral efficiency is given by

$$
S_{e, A R Q}=\frac{S_{e, P H Y}}{\bar{N}\left(P_{e}, R^{\max }\right)}=\frac{1-P_{e}}{\left(1-P_{e}^{R^{\max }+1}\right)(N+1)} \sum_{n=1}^{T} R_{n} P_{n}
$$

Setting $R^{\max }=0$ in (13), we obtain (11) which corresponds to the average spectral efficiency for AMC only. Moreover probability of the actual packet loss at the data link layer with the $R^{\max }$ truncated ARQ is $P_{e}^{R^{\max }+1} \leq P_{\text {target }}^{R^{\max }+1}=P_{\text {loss }}$ that satisfies both delay and error performance constraint.

\subsection{Joint-OPTIMIZation OF AMC With AdAPTIVE R ${ }^{\mathrm{MAX}}$ AND N}

The discussions in the previous sections implicitly assumed that the values for $N$ and $R^{\max }$ are fixed. The optimal joint-adaptation of AMC transmission mode with an adaptive number of cooperating relays $N$ and adaptive maximum retransmission limit $R^{\max }$ can be performed by finding the optimal set $\left[N^{*}, R^{\max }\right]$ for every mean received SNR that maximizes (13) while satisfying (3). Now, we have developed an algorithm that can be used to achieve the optimized parameters in a myriad of fading environments by following below four steps: 
Step 1: Specify a range of integer values for both $N$ and $R^{\max }$. In this paper, we have arbitrarily chosen $N=[0,1,2,3,4]$ and $R^{\max }=[0,1,2,3, \ldots, 10]$.

Step 2: For a specified packet loss probability $P_{\text {loss }}$ (arbitrarily chosen to be 0.01 ) constraint at the data link layer, determine $P_{\text {target }}$ (i.e., error constraint at the PHY layer) for each value of $R^{\max }$ using (3).

Step 3: From Step 2, we obtain a set of $\left[R^{\max }, P_{\text {target }}\right]$ pairs. For each value of the $P_{\text {target }}$ found in Step 2, find the switching SNR thresholds for AMC using (5).

Step 4: At each mean channel SNR, evaluate the average spectral efficiency for each set of $[N$, $\left.R^{\max }, P_{\text {target }}\right]$ using (13).

The optimal $\left[N^{*}, R^{\max }\right]$ is then simply the set that yields the highest throughput.

To the best of our knowledge a similar approach which focuses on throughput optimization by jointly adaptation of both $N$ and $R^{\max }$ has not been considered in the previous literature.

\section{NUMERICAL RESULTS}

In this section, numerical findings are presented for the APER, average spectral efficiency and outage probability of the link-adaptive CAF relay networks using our proposed analytical frameworks.

In this section, below fading severity parameters and average link SNRs (arbitrarily chosen) will be utilized to generate the plots (unless stated otherwise): $m_{s, d}=1, m_{s, 1}=m_{1, d}=m_{s, 2}=m_{2, d}$ $=m_{s, 3}=m_{3, d}=m_{s, 4}=m_{4, d}=2, \Omega_{s, d}=0.2 \mathrm{Es} / \mathrm{N} 0, \Omega_{s, 1}=\Omega_{s, 3}=\Omega_{2, d}=\Omega_{4, d}=E_{s} / N_{0}, \Omega_{s, 2}=0.86 E_{s} / N_{0}, \Omega_{s, 4}=\Omega_{3, d}$ $=0.8 E_{s} / N_{0}, \Omega_{1, d}=0.5 E_{s} / N_{0}$; where $m_{a, b}$, denotes the fading index of link $a-b$. We have also assume $P_{\text {loss }}=0.01$. To generate all the plots we used MGF of minimum SNR bound $\tilde{\gamma}_{i}=\min \left(\gamma_{s, i}, \gamma_{i, d}\right)$ for two-hop relayed path over Nakagami-m channel with i.n.d fading statistics, and is given by [23],[25]

$$
\phi_{\bar{\gamma}_{i}}^{(U B)}(s)=\sum_{\substack{k \in\{(s, i)(i, d)\} \\ j \neq k}} \frac{\Gamma\left(m_{k}+m_{j}\right)}{m_{k} \Gamma\left(m_{k}\right) \Gamma\left(m_{j}\right)}\left(\frac{\Omega_{j} m_{k}}{s \Omega_{j} \Omega_{k}+\Omega_{j} m_{k}+\Omega_{k} m_{j}}\right)^{m_{k}}{ }_{2} F_{1}\left(1-m_{j}, m_{k} ; 1+m_{k} ; \frac{\left(s \Omega_{k}+m_{k}\right) \Omega_{j}}{s \Omega_{j} \Omega_{k}+\Omega_{j} m_{k}+\Omega_{k} m_{j}}\right)
$$

in conjunction with (2). Whereas, MGF for the lower bound may be computed as $\phi_{\hat{\gamma}_{i}}^{(L B)}(s / 2)$. In (14), $m_{q}$ is the Nakagami-m fading parameter and $\Omega_{q}=E\left[\gamma_{q}\right]$ corresponds to the mean link SNR. 
International Journal of Computer Networks \& Communications (IJCNC) Vol.8, No.3, May 2016

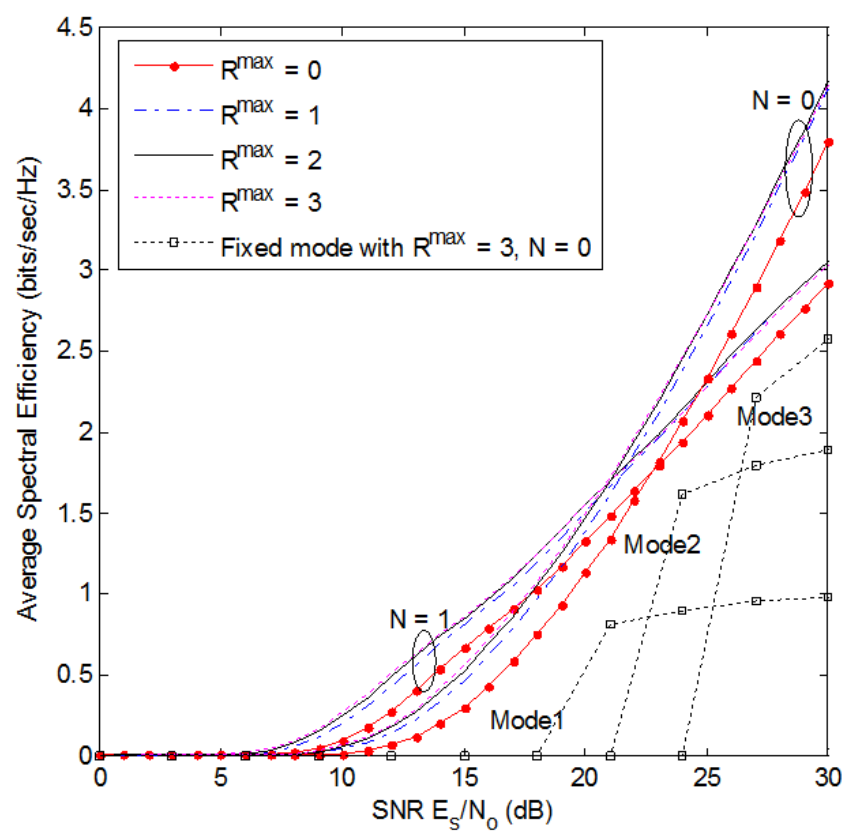

Fig. 3 Mean spectral efficiencies of non-cooperative and CAF relay networks for TM1 mode.

Fig. 3 and Fig. 4 depict the mean spectral efficiency and APER for both non-cooperative $(N=0)$ and cooperative amplify-and-forward $(N=1)$ wireless relay networks as a function of $E_{s} / N_{0}$ for different values of retransmission, $R^{\max }$. Moreover, to show the comparison with our proposed analysis, plots of fixed modes with $R^{\mathrm{max}}=3, \mathrm{~N}=0$ are generated using [10, eq. (17)] in Fig 3. It is clear that combining AMC with truncated ARQ for CAF relay networks offers much higher spectral efficiency than the truncated ARQ with fixed modulation for non-diversity system.

It is apparent that the average spectral efficiency curves for the joint-design of AM with truncated ARQ are significantly greater than the AM case only (i.e., when $R^{\max }=0$ ), as expected. Although increasing $R^{\max }$ can potentially yield a higher mean spectral efficiency, however, this improvement is achieved at the cost of increased APER (refer Fig. 4). When $R^{\max }$ is increased, the ARQ protocol (at the data link layer) assumes greater responsibility for assuring the required packet delivery performance and relieves the Physical layer from the rigorous error control requirement, thereby enabling higher rates (i.e., larger constellation size) at the Physical layer while satisfying the packet loss constraint at the MAC layer. But it is also important to note that $\bar{N}\left(P_{e}, R^{\max }\right)$ increases with $R^{\max }$. Therefore, we expect that the overall spectral efficiency increase initially with increasing $R^{\max }$ but declines beyond some optimum $R^{\max }$ value especially at higher values of $E_{s} / N_{0}$. Moreover, not obvious from Fig. 3, we observed that the mean spectral efficiency of the CAF network with cross-layer combining of AM and truncated ARQ increases initially with the increasing $R^{\max }$ but declines beyond some optimum $R^{\max }$ value (see Fig. 5), this motivated us to study the efficacy of an adaptive $R^{\max }$ strategy for delay insensitive applications in Fig 6. 
International Journal of Computer Networks \& Communications (IJCNC) Vol.8, No.3, May 2016

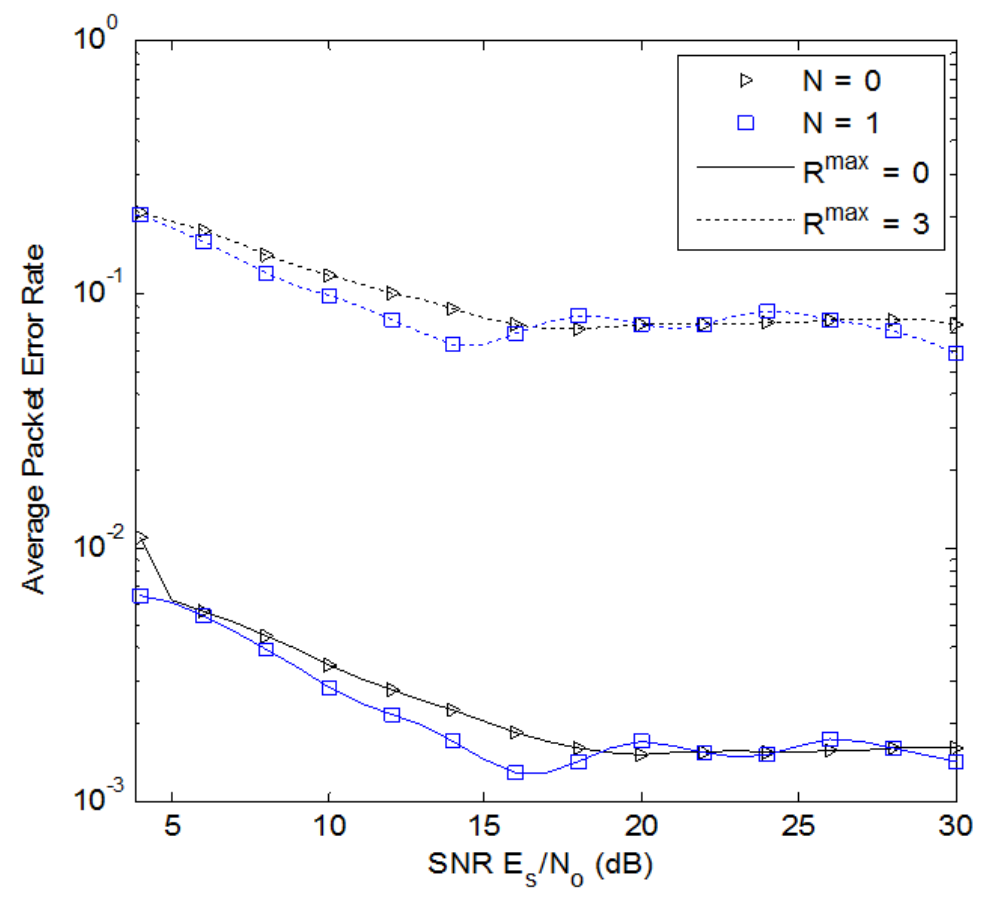

Fig. 4 APER (at the PHY layer) performance of both non-cooperative and CAF relay networks for TM1.

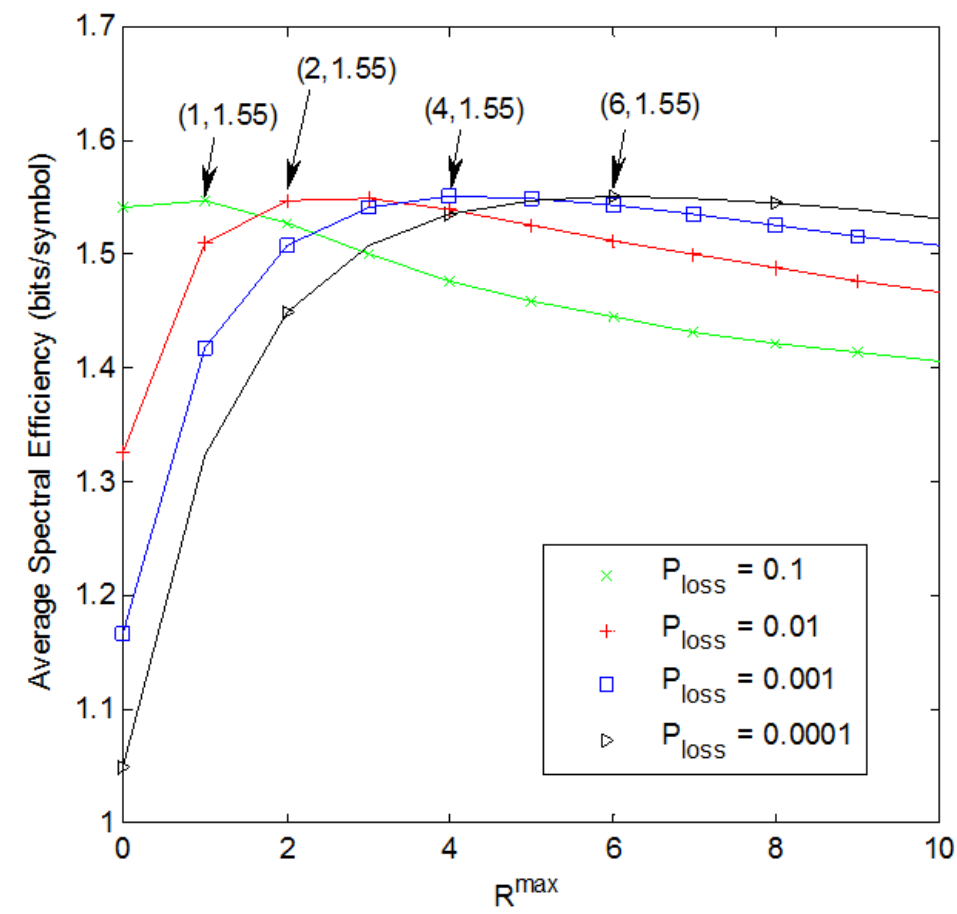

Fig. 5 Mean spectral efficiencies of a CAF relay network as a function of $R^{\max }$ (at fixed $\mathrm{E}_{\mathrm{s}} / \mathrm{N}_{\mathrm{o}}=20 \mathrm{~dB}$ ) for TM1 mode. 
International Journal of Computer Networks \& Communications (IJCNC) Vol.8, No.3, May 2016

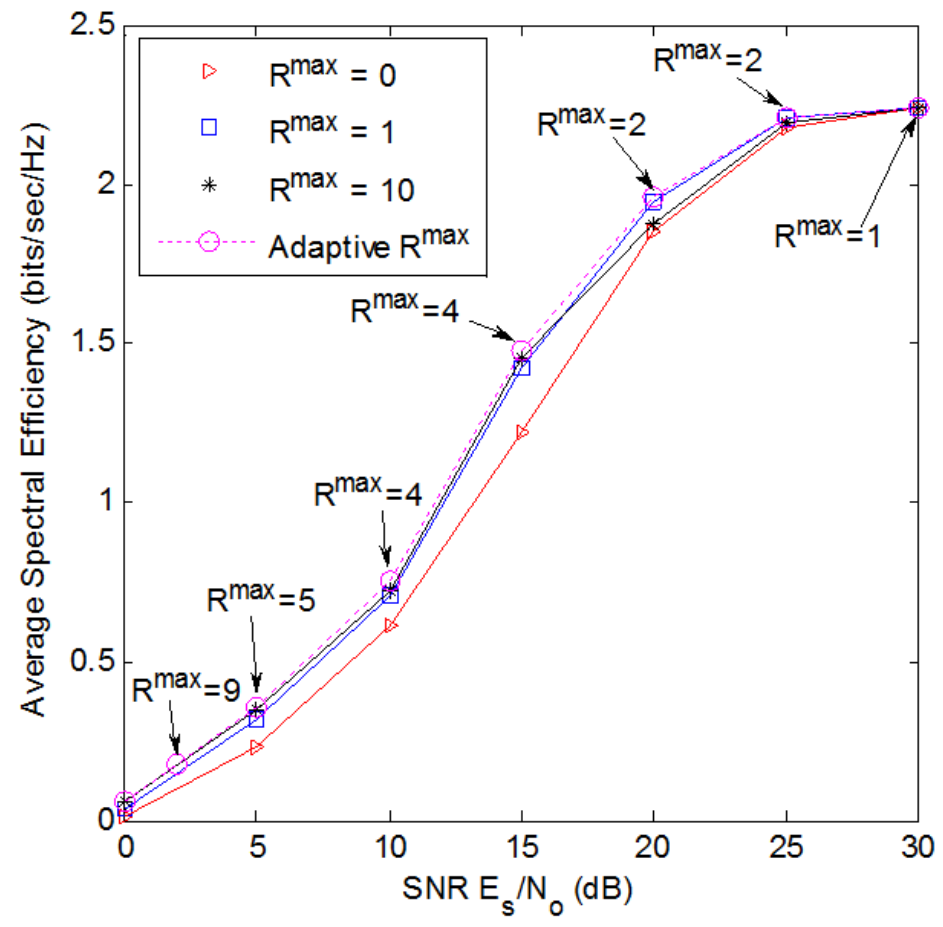

Figure 6 A comparison between our proposed adaptive $\mathrm{R}^{\max }$ strategy with the traditional fixed $\mathrm{R}^{\max }$ truncated ARQ scheme in a CAF relay network $(N=1)$ for TM2 mode

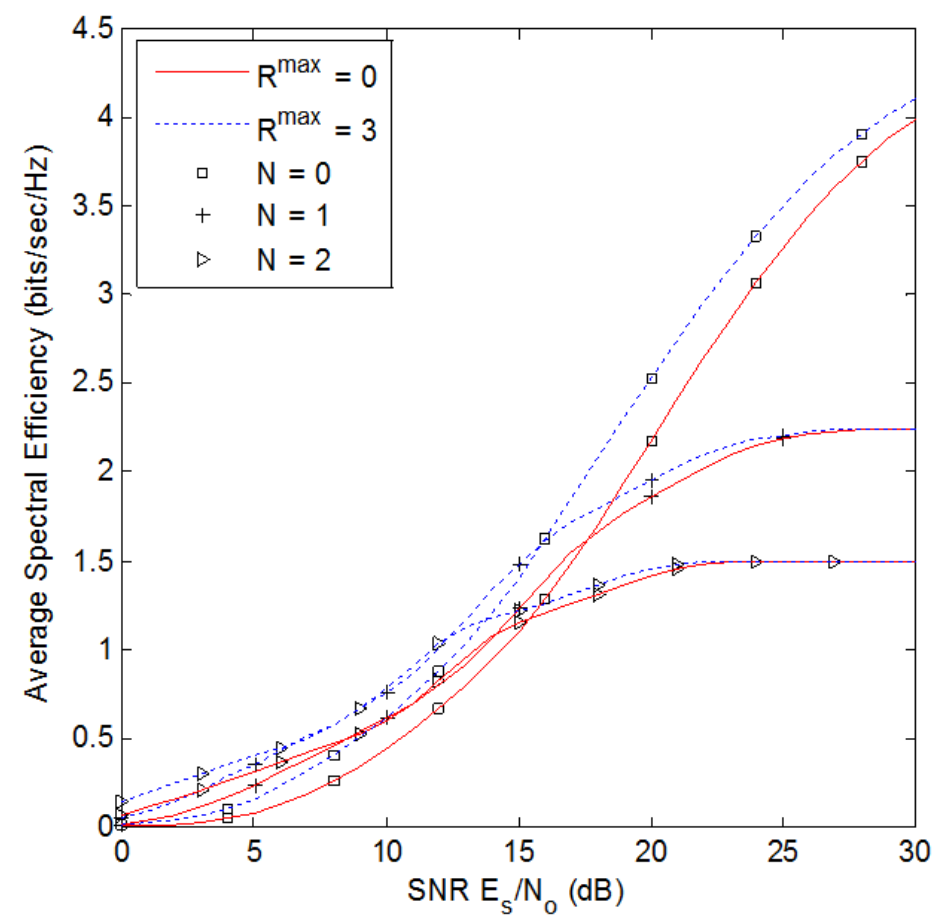

Figure 7 Average Spectral Efficiency vs. average SNR consisting of $N$ relays $(\mathrm{N}=0,1,2)$ for TM2 mode. 
Fig. 7 depicts the mean spectral efficiencies of CAF and non-cooperative $(N=0)$ networks that employ AMC with TM2 mode. It is evident that adapting $N$ (the number of collaborating relay nodes) to the prevailing channel conditions is an effective strategy to dramatically increase the average spectral efficiency in tactical-edge (low/moderate SNR) environments, while satisfying the prescribed delay and packet loss constraints. Moreover, the average spectral efficiency of CAF relay network is considerably higher than the non-cooperative system at low and moderate SNRs. Although CAF system can utilize the inherent spatial diversity scheme in wireless broadcast transmissions, there is a loss in spectral efficiency due to its half-duplex operation [2324]. In fact, there is no incentive in using cooperative diversity when the $S-D$ link is good. This observation in turn suggests that we should adapt $N$ to the prevailing channel conditions (i.e., increasing $N$ as the channel condition deteriorates to provide additional diversity and maximize the average spectral efficiency while satisfying the prescribed delay and packet loss constraints). There exists an optimum $N$ that maximizes the mean spectral efficiency for a specified SNR. Thus, the observations in Figs. 3, 5 and 6, motivated us to study the efficacy of joint-adaptation of $\left[N^{*}, R^{\max }\right.$ ] for CAF relay networks with AMC at the PHY layer in Fig 8.

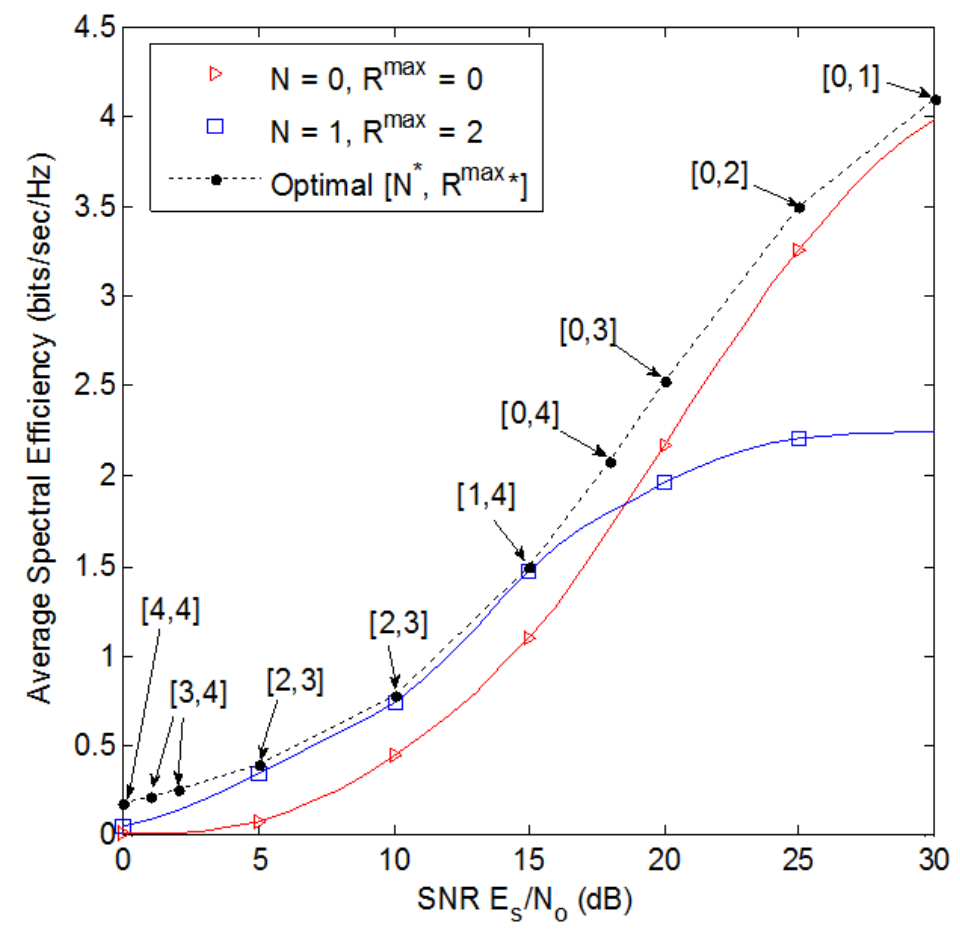

Figure 8 Mean spectral efficiencies of CAF relay networks with/without joint optimization of $\left[N^{*}, \mathrm{R}^{\mathrm{max} *}\right]$ in conjunction with AMC TM2 mode.

In Fig. 8, we examine the effectiveness of joint-adaptation of $\left[N^{*}, R^{\max *}\right]$ for CAF relay networks with AMC at the PHY layer. It is important to note compared to our previous results in Fig. 6 these results provide a different perspective on the system analysis, where we considered only adaptive $R^{\max }$ scheme (which is a single parameter adaptation with fixed number of relays). In this we introduced an interesting approach for maximization of throughput using joint adaptation of two parameters, one with the cooperative diversity order $N$ and second with the adaptive $R^{\max }$ scheme. The curve corresponding to the "optimal" case is generated using the algorithm highlighted in Section III.F. It is apparent that our anticipated adaptive CAF system (i.e., AMC with adaptive $N$ and $R^{\max }$ ) achieves significantly higher average spectral efficiency than the noncooperative wireless system with AMC only $\left(N=0, R^{\max }=0\right)$ particularly at low and moderate 
values of $E_{s} / N_{0}$. Besides, the joint-optimization of $\left[N^{*}, R^{\max *]}\right.$ not only maximizes the mean spectral efficiency at low mean SNRs, but it is also reduces the average delay experienced with $R^{\max }$ adaptation alone as in Fig 6 . This shows that the optimization of $\mathrm{N}$ is very critical on system performance compared to the $R^{\max }$.

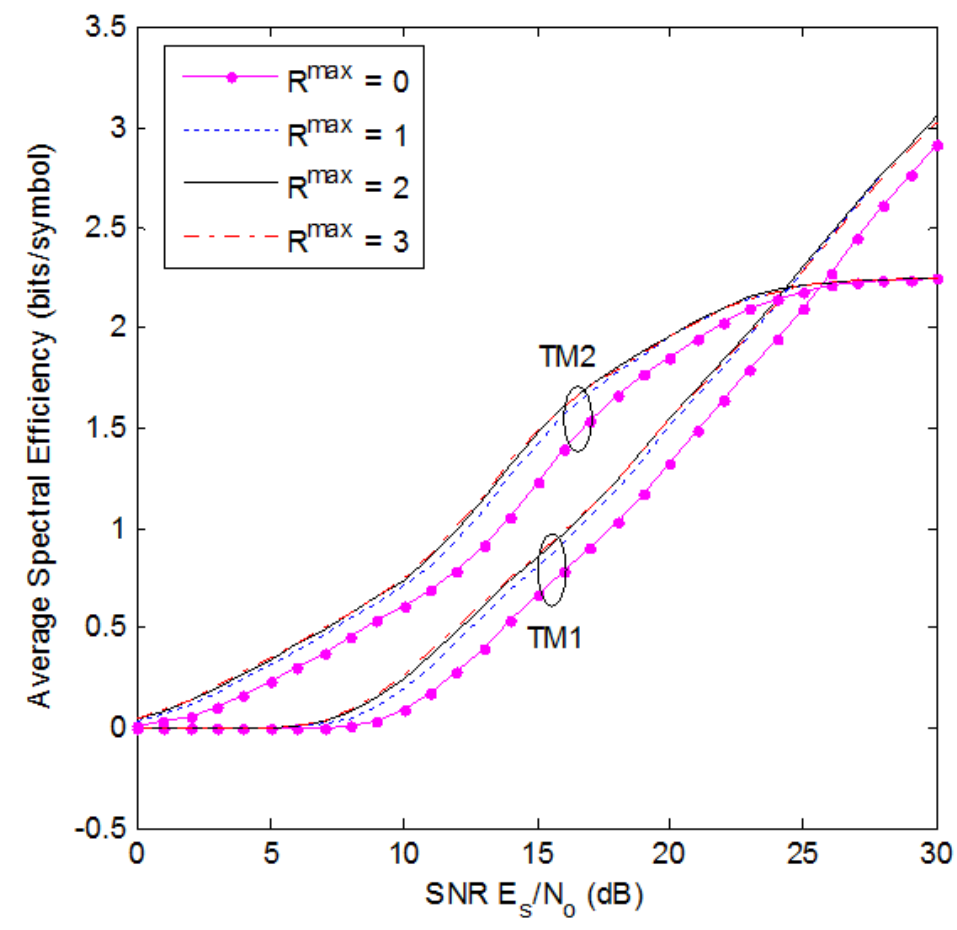

Figure 9 Average Spectral Efficiency vs. average SNR consisting of 1 relay for TM1 and TM2 mode.

Fig 9 shows the spectral efficiency comparison of AMC TM1 and TM2 modes for a CAF relay network with a single cooperating relay. It is evident that AMC TM2 mode outperforms TM1 mode at low and moderate values of $E_{S} / N_{0}$, which can be attributed to the FEC advantage at the PHY layer (coding gain). However, at high values of $E_{S} / N_{0}$, an opposite trend is observed. This is because the corresponding modes in TM1 support higher data rates (i.e., the highest rate mode has 7 bits/symbol in TM1 which is much larger than 4.5 bits/symbol in TM2). It is also apparent from Fig. 9 that the relative difference between the spectral efficiency curves is greatest when $R^{\max }$ is increased from 0 to 1 . This shows that joint PHY/MAC design with smaller values of $R^{\max }$ can achieve sufficient spectral efficiency gain (i.e., it is attractive from a practical stand-point, given that they incur smaller delays and packet buffer requirements) although the optimum $R^{\max }$ rises exponentially with the decreasing values of $E_{S} / N_{0}$ (see Fig. 6). 


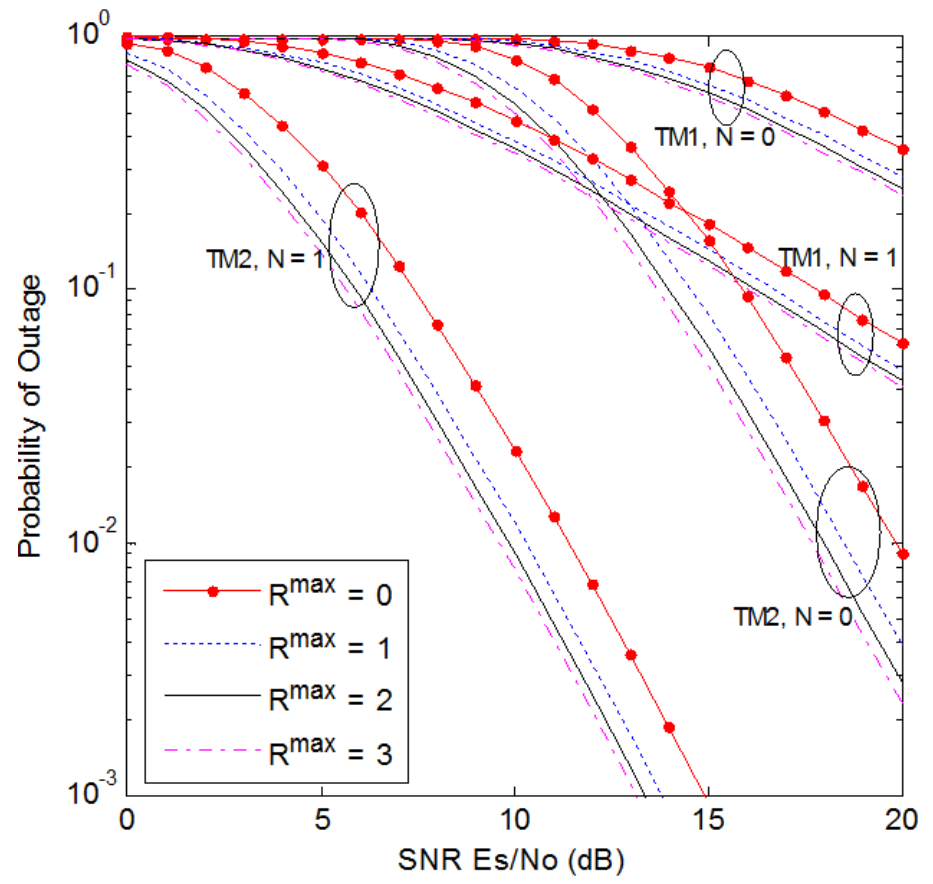

Figure 10. Probability of outage vs. average SNR consisting of $N$ relays $(\mathrm{N}=0,1)$ for TM1 and TM2 mode.

Fig. 10 depicts the probability of outage (i.e., probability that source node ceases transmission because the prescribed target PER cannot be satisfied) performance curves for link-adaptive noncooperative/CAF relay networks. It is evident that the CAF relay network $(N=1)$ outperforms the direct transmission case $(N=0)$ since the former exploits the available "user cooperation" (spatial) diversity gain. Similarly, AMC TM2 mode exhibits better performance than the TM1 mode due to coding gain. A larger $R^{\max }$ also translates into a lower $P_{\text {out }}$ because of the increased time-diversity order.

\section{Conclusions}

In this paper we analyzed and examined the efficiency of a join-design of adaptive modulation and coding (AMC) at the physical layer with an adaptive $R^{\max }$-truncated selective-repeat automatic repeat request (ARQ) protocol at the data link layer to maximize the throughput of the cooperative non-regenerative relay networks under prescribed delay and/or error performance constraints. In particular, we generalize the existing design/results for cross-layer combining of $\mathrm{AMC}$ at physical layer with truncated ARQ at data link layer with non-cooperative diversity systems in three-folds: (i) extension of the existing cross-layer PHY/MAC design with noncooperative model to cooperative diversity model; (ii) generalization/unification of mathematical expressions for various network performance metrics to generalized block fading channels with independent but non-identically distributed (i.n.d) fading statistics among the spatially distributed nodes; (iii) analysis of the effectiveness of joint-adaptation of the maximum retransmission limit $R^{\max }$ in ARQ protocol and cooperative diversity order $N$ for delay-insensitive applications. Our intuitive numerical outcomes reveal that the average throughput can be increased significantly by judiciously combining two additional degrees of freedom (i.e., cooperative diversity order $N$ and retransmission limit $R^{\max }$ ) that are available in CAF relay networks besides employing AMC at the PHY layer, particularly in the most challenging practical scenario of low signal-to-noise ratio (SNR) regime. 
International Journal of Computer Networks \& Communications (IJCNC) Vol.8, No.3, May 2016

\section{ACKNOWLEDGEMENT}

This research work is supported in part by funding from the US Air Force Research Laboratory/Clarkson Aerospace, US Army Research Office and the National Science Foundation.

\section{REFERENCES}

[1] M. Alouini and A. Goldsmith (2000), “Adaptive modulation over Nakagami fading Channel," Kluwer J. Wireless Commun., vol. 13, no. 1-2 pp. 119-143.

[2] D. L. Goeckel, (1999) "Adaptive Coding for Time-Varying Channels using Outdated Fading Estimates," IEEE Trans. Commun., vol. 47, pp. 844-855.

[3] A. Goldsmith and S. Chua, (1998) "Adaptive Coded Modulation for Fading Channels," IEEE Trans. Commun., vol. 46, pp. 595-602.

[4] E. Malkamaki and H. Leib, (2000) "Performance of Truncated type-II hybrid ARQ schemes with Noisy Feedback over Block Fading Channels," IEEE Trans. Commun., vol. 48, pp. 1477-1487.

[5] Weifeng, Su., Ahmed K. S., \& Ray Liu K. J, (2008) "Cooperative Communication Protocols in Wireless Networks: Performance Analysis and Optimum Power Allocation,” Springer link Wireless Personal Communication, vol. 44, pp. 181-217.

[6] T. Nechiporenko, K. Phan, C. Tellambura and H. Nguyen, (2008) "Performance Analysis of Adaptive M-QAM for Rayleigh Fading Cooperative Systems," Proc. IEEE International Conference on Communication, pp. 3393-3399.

[7] A. Annamalai, B. Modi, and R. Palat, (2011) "Analysis of Amplify-and-Forward Cooperative Relaying with Adaptive Modulation in Nakagami-m Fading Channels," Proc. IEEE Consumer Communication and Networking Conf., pp. 1116-1117.

[8] B. Modi, A.Annamalai and R.Palat (2011) "An MGF Approach for Performance Evaluation of NonRegenerative Cooperative Relay Networks with Adaptive Modulation in Nakagami-m Fading Environments," IEEE Wireless Telecommunications Symposium, pp. 1-6.

[9] Bhuvan Modi and A. Annamalai, (2011) "Improving the Spectral Efficiency of Amplify-and-Forward Cooperative Relay Network with Adaptive M-QAM Modulation," IEEE 20th International Conference on Computer Communications and Networks, pp. 1-6.

[10] Q. Liu, S. Zhou, and G. Giannakis, (2004) "Cross-Layer Combining of Adaptive Modulation and Coding with Truncated ARQ over Wireless Links,” IEEE Trans. Wireless Comm., vol.3, pp. 17461755.

[11] L. Dai and K. B. Letaief, (2005) "Cross-Layer Design for Combining Cooperative Diversity with Truncated ARQ in Ad-hoc Wireless Networks," IEEE GLOBECOM Telecomm. Conf., pp. 31793183.

[12] J. Harsini and M. Zorzi, (2011) "Effective Capacity Analysis for Multi-Rate Relay Channels Exploiting Adaptive Cooperative Diversity," IEEE International Conf., on Comm., pp.1-6.

[13] M. Mardani, J. Harsini, F. Lahouti and B. Eliasi, (2011) "Link Adaptive and QoS Provisioning Cooperative ARQ-Applications to Relay Assisted Land Mobile Satellite Communications," IEEE Trans. Vehicular Tech., vol. 60 pp. 3192-3206,

[14] F. Shi and D. Yuan, (2008) "Cross-Layer Combination of Cooperative HARQ with AMC in Wireless Ad-hoc Networks,” IEEE international Conf. on Commu. Sys., pp.896-900.

[15] R. C. Palat, A. Annamalai, and J. H. Reed, (2008) "An Efficient Method for Evaluating Information Outage Probability and Ergodic Capacity of OSTBC Systems,” IEEE Comm. Letters, vol. 12, pp. 191-193.

[16] B. Modi, A. Annamalai, O. Olabiyi, and R. Chembil Palat, (2012) "Ergodic Capacity Analyses of Cooperative Amplify and Forward Relay Networks over Rice and Nakagami Fading Channels," International Journal of Wireless and Mobile Networks, vol4, no. 1, pp. 97-116.

[17] N. Laneman, D. Tse and G. Wornell (2004) "Cooperative Diversity in Wireless Networks: Efficient Protocols and Outage Behaviour," IEEE Trans. Information Theory, vol. 50, pp. 3062-3080.

[18] M. Hasna and M. Alouini (2004) "Harmonic Mean and End-to-End Performance of Transmission System with Relays,” IEEE Trans. Communications, vol. 52, no.1, pp. 130-135.

[19] O. Olabiyi and A. Annamalai, (2011) "ASER Analysis of Cooperative Non-Regenerative Relay Systems over Generalized Fading Channels" IEEE International Conf. on Comp. Commu. and Networks, pp.1-6. 
[20] A. Annamalai, B. Modi, R. Palat and J. Matyjas, (2010) "Tight-Bounds on the Ergodic Capacity of Cooperative Analog Relaying with Adaptive Source Transmission Techniques," IEEE International Symp. on Personal, Indoor, and Mobile Radio Comm., pp.18-23.

[21] R. Mostafa, A. Annamalai, and H. Reed, (2004) "Performance Evaluation of Cellular Mobile Radio System with Interference Nulling of Dominant Interferers,” IEEE Trans. Commun., vol. 52, pp.326335.

[22] S. B. Wicker, (1995) Error Control Systems for Digital Communication and Storage. Englewood Cliffs, NJ: Prentice-Hall.

[23] A. Annamalai, R. Palat, and J. Matyjas (2010) "Estimating Ergodic Capacity of Cooperative Analog Relaying under Different Adaptive Source Transmission Techniques," Proc. IEEE Sarnoff Symposium, pp.1-5.

[24] B. Modi, and A. Annamalai, (2011) “ Improving the Spectral Efficiency of Amplify-and- Forward Cooperative Relay Network with Adaptive M-QAM Modulation," Proc. IEEE International Conference on Computer Communications and Networks, pp. 1-6.

[25] B. Modi, A. Annamalai, O. Olabiyi and R. Palat, (2013) "Ergodic capacity analysis of cooperative amplify-and-forward relay networks over generalized fading channels", Wiley Journal of Wireless and Mobile Computing, vol. 15, no. 8, pp.1259-1273.

\section{Authors}

Dr. Bhuvan Modi received PhD. degree from Prairie View A \& M University, Texas A \& M University System, in 2012. He earned his M.S. degree in Electrical Engineering from Lamar University, United States of America, M.S. degree in Electronics and Communication Engineering from Dharmsinh Desai University, India, and the B.S. degree in Electronics and Communication Engineering from North Gujarat University, India, in 2009, 2002 and 2001, respectively. He is currently working as a Senior

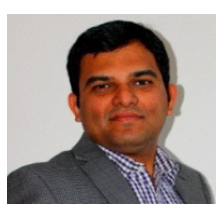
Member of Technical Staff at AT\&T Mobility Lab Seattle, WA. Currently Dr. Modi serves as an editorial committee//International editorial board member for journals and organizations, namely the International Journal of Wireless and Mobile Networks (IJWMN), The Standard International Journals (SIJ), the International Journal of Wireless and Mobile Communication for Industrial Systems, Science \& Engineering Research Support Society, and First International Workshop on Wireless and Mobile Communication for Industrial Systems (WMCIS 2015) and has also been invited to serve on the international editorial board committee member for the Journal of Advanced Research in Wireless, Mobile \& Telecommunication. He received 'Student Travel Grant Award' to present his work at the IEEE MILCOM'11 and achieved excellent work appreciation certificate award from Vice President and CEO, AT\&T Mobility Lab for individual contribution towards successfully launch of WiFi Calling Service in the US Market. Over the last few years, Dr. Modi has published over a dozen peer reviewed conference and journal articles. His current research interests include cross-layer design/optimization for adaptive-link cooperative relay networks, 4G/5G Wireless Technologies, Openstack and software-defined radios.

Dr. Oluwatobi Olabiyi received the B.Sc. degree in Electronic and Electrical Engineering from Obafemi Awolowo University, Ile-Ife and M.S. and $\mathrm{PhD}$ degrees in Electrical Engineering Prairie View A\&M University, Texas. Over the last three years, he has co-authored approximately two-dozen peer-reviewed conference and journal articles. He was the recipient of the Roy G. Perry College of Engineering Outstanding Masters Student of the Year Award (2011) and the National Society of Black

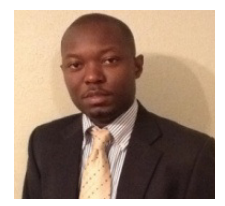
Engineer's Golden Torch Award for Graduate Student of Year (2012). His research interests include dynamic spectrum access, MIMO, cooperative communications, statistical signal processing, compressive sensing, machine-learning and optimization techniques. 
Dr. Annamalai is presently the Director of Center of Excellence for Communication Systems Technology Research, a Texas A\&M Board of Regents approved University Research Center at the Prairie View A\&M University, and a Professor in the Department of Electrical and Computer Engineering. He has over 16 years of research/teaching experience in wireless communications at Motorola, University of Victoria, Air Force Research Laboratory, Virginia Tech and PVAMU with approximately 200 peer-reviewed publications and 5 book chapters. Dr. Annamalai has been honored by his colleagues on

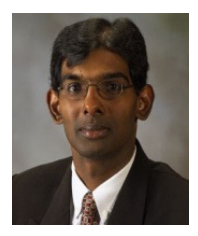
numerous occasions for his excellence in research including winning the 2011 Roy G. Perry College of Engineering Outstanding Faculty (Research) Award, IEEE Leon Kirchmayer Prize Paper award, ASEE/AFOSR Summer Faculty Fellowships, NSERC Doctoral Prize, CAGS/UMI Distinguished Doctoral Dissertation Award, IEEE VTS/Motorola Daniel E. Noble Fellowship, among others. He had served on the Editorial Boards of four IEEE journals/transactions in the last 12 years, and has helped to organize a few major IEEE conferences on wireless communications including serving in the capacity of Technical Program Chair of the 2002 IEEE Vehicular Technology Conference in Vancouver, Canada. His current research interests include cooperative spectrum sensing, compressive sensing, cross-layer design for scalable multimedia transmission and cooperative wireless communications. 